\title{
Research for Equalizing of the Illumination of the Underwater Image Using the Artificial Illumination
}

\author{
Masato Shinto, ${ }^{\mathrm{a},}$, Qinqin Honga ${ }^{\mathrm{a}}$, Meng Gea, Lifeng Zhang ${ }^{\mathrm{a}}$ \\ a'1-1sensui-cho Tobataku, Kitakyushu city, Fukuoka Japan 804-8550 \\ Kyushu Institute of Technology \\ *Corresponding Author: shinto.masato916@mail.kyutech.jp
}

\begin{abstract}
The deep sea image has a feature that the luminance balance is not flat because the lighting beam direction. This made the original took image has a poor visual impress. This paper proposed a new approach to improve visibility of deep sea image with difference in luminance by modifying the DCT coefficients in the low frequency area. By using DCT, pixel values in space domain of an image are converted into a frequency domain and most of the energy is concentrated in a low frequency domain. Human eyes are sensitive to changes in low frequency components. Therefore, by changing the low frequency component, it is possible to create an image for human beings to see easily. The simulation shows a reasonable result.
\end{abstract}

Keywords: DCT coefficients, Gaussian filter, luminance.

\section{Introduction}

Japan is a resource absent country, many of the mine resource depends on import. Nowaday, the mine insufficient happened globally, therefore the Japanese government start the project to detect the submarine mine around the country sea. In recent years it has been confirmed that abundant resources exist in the ocean floor in Japan's economic watershed. For example, there are submarine hydrothermal deposits, methane hydrate, cobalt crust, and so on. Furthermore, in order to adapt to harsh environmental conditions such as high water pressure, low water temperature, darkness and low oxygen condition, organisms have evolved independently, and there is a unique form / ecology that can't be imagined from the mesopelagic species.

The deep sea generally refers to a place with a water depth of $200 \mathrm{~m}$ or less. This proportion accounts for approximately $95 \%$ of the ocean. Fine particles and water molecules present in water have the property of absorbing sunlight. The energy of the sun's light is about $0.1 \%$ of the surface of the sea near the water depth of $200 \mathrm{~m}$, and general phytoplankton is said to be the limit of photosynthesis. So, when the water depth exceeds $200 \mathrm{~m}$, the sight becomes dark and human eyes can't recognize the object. Therefore, artificial lighting is indispensable in deep sea surveys. Normally, the illumination is directed to the target object. There are creatures which avoid the light so it is necessary to reduce luminous intensity. Bright parts and dark parts are generated in the image because the illuminance is decreased. Then, using the image processing, reduce the bright and dark areas in the image

\section{Principle}

\section{$2.1 \quad \mathrm{YCbCr}$}

The color is expressed in three primary colors of light of RGB (red, blue, yellow). Human eyes are sensitive to changes in brightness, but they are insensitive to color change. Therefore, in the current image technology, image processing is carried out by decomposing colors into $\mathrm{YCbCr}$ (luminance, hue / saturation of red system, hue / saturation of blue system) components. So convert the input RGB component to the $\mathrm{YCbCr}$ component in order to equalize the illuminance of the whole image. 
In a general color image, one pixel is represented by a total of 24 bits of 8 bits ( 0 to 255 ) of R, G and B. When these are converted to $\mathrm{YCbCr}$, they take the following values.

$$
\begin{aligned}
& \mathrm{Y}=0.299 \mathrm{R}+0.587 \mathrm{G}+0.114 \mathrm{~B} \\
& \mathrm{Cb}=(\mathrm{B}-\mathrm{Y}) 0.564+\text { delta } \\
& \mathrm{Cr}=(\mathrm{R}-\mathrm{Y}) 0.713+\text { delta }
\end{aligned}
$$

RGB is the value of $\mathrm{R}, \mathrm{G}, \mathrm{B}$ of each pixel, and delta is categorized as follows.

$$
\text { delta }=\left\{\begin{array}{c}
128 \text { for } 8 \text { bit images } \\
32768 \text { for } 16 \text { bit images } \\
0.5 \text { for floating point images }
\end{array}\right.
$$

\subsection{DCT(Discrete Cosine Transform)}

DCT (Discrete Fourier Transform) is a method of converting discrete signals to frequency domain. DCT transforms a finite number sequence into coefficients of a linear combination based on a cosine function number sequence. The cosine function returns real number if the input is real number. In addition, there is a property that information concentrates on low frequency components. It is suitable for data compression because information on high frequency components is difficult for humans to recognize. IDCT (Inverse Discrete Fourier Transform) is a method of converting frequency domain to discrete signals. DCT and IDCT are expressed by the following formulas.

$$
\mathrm{F}(\mathrm{i}, \mathrm{j})=\mathrm{C}(i) \mathrm{C}(\mathrm{j}) *
$$

$\sum_{x=0}^{M-1} \sum_{y=0}^{N-1} f(x, y) \cos \frac{(2 x+1) i \pi}{2 M} \cos \frac{(2 y+1) j \pi}{2 N}$

$$
\mathrm{f}(\mathrm{x}, \mathrm{y})=\frac{4}{M N} *
$$

$\sum_{i=0}^{M-1} \sum_{j=0}^{N-1} \mathrm{C}(i) \mathrm{C}(\mathrm{j}) F(i, j) \cos \frac{(2 x+1) i \pi}{2 M} \cos \frac{(2 y+1) j \pi}{2 N}$

$f(x, y)$ : Two dimensional discrete signal value of input image $(x=0,1, \cdots M-1, y=0,1, \cdots N-1)$

$F(i, j)$ : Two dimensional DCT coefficient value after DCT $(\mathrm{i}=0,1, \cdots \mathrm{M}-1, \mathrm{j}=0,1, \cdots \mathrm{N}-1)$

$\mathrm{M}$ is the height of the image and $\mathrm{N}$ is the width of the image.

$$
\mathrm{C}(\mathrm{p})=\left\{\begin{array}{c}
\frac{1}{\sqrt{2}}(p=0) \\
1 \quad(p \neq 0)
\end{array}\right.
$$

\subsection{Gaussian Filter}

In the moving average filter, the target pixel is placed in the center of an odd square type kernel with one side of 3 or more. Next the pixel value in the square divides by the square number of length of one side. Finally, the pixel values are averaged by returning their sum to the target pixel. In many cases, the pixel value close to the pixel of interest is approximately equal to the pixel value of the pixel of interest, and the difference from the pixel value of the pixel of interest increases as the distance from the pixel of interest increases. Taking this into consideration, it is the Gaussian filter that the weight at the time of calculation is made larger as closer to the pixel of interest, and the weight is made smaller as it gets farther. This filter uses the following Gaussian distribution function.

$$
\mathrm{g}(\mathrm{x}, \mathrm{y})=\frac{1}{2 \pi \sigma^{2}} \exp \left(-\frac{x^{2}+y^{2}}{2 \sigma^{2}}\right)
$$

$\mathrm{g}(\mathrm{x}, \mathrm{y})$ : Two dimensional discrete signal value of input image

$\sigma \quad$ : Standard deviation

$\sigma^{2}:$ Dispersion

\section{Experimental Method}

\subsection{Proposed method}

Underwater image converts to $\mathrm{YCbCr}$ and sets the image of $\mathrm{Y}$ component as the original image. Do the DCT conversion and save the DC component at the upper left end. Using several shapes to set the value of the DCT coefficient in that region to zero. The shape to be used is shown in Fig.1. The DCT coefficient of the white area in Fig. 1 is set to 0 . Substitute the stored DC component for the DCT coefficient at the upper left corner. Do the IDCT conversion.

\subsection{Conventional Method}

Apply a Gaussian filter to the original image and create luminance distribution of image. An image of luminance distribution is shown in Fig.2. Take the difference in luminance distribution created from the original image. With this method, depending on the image, it is necessary to make the luminance distribution many times 

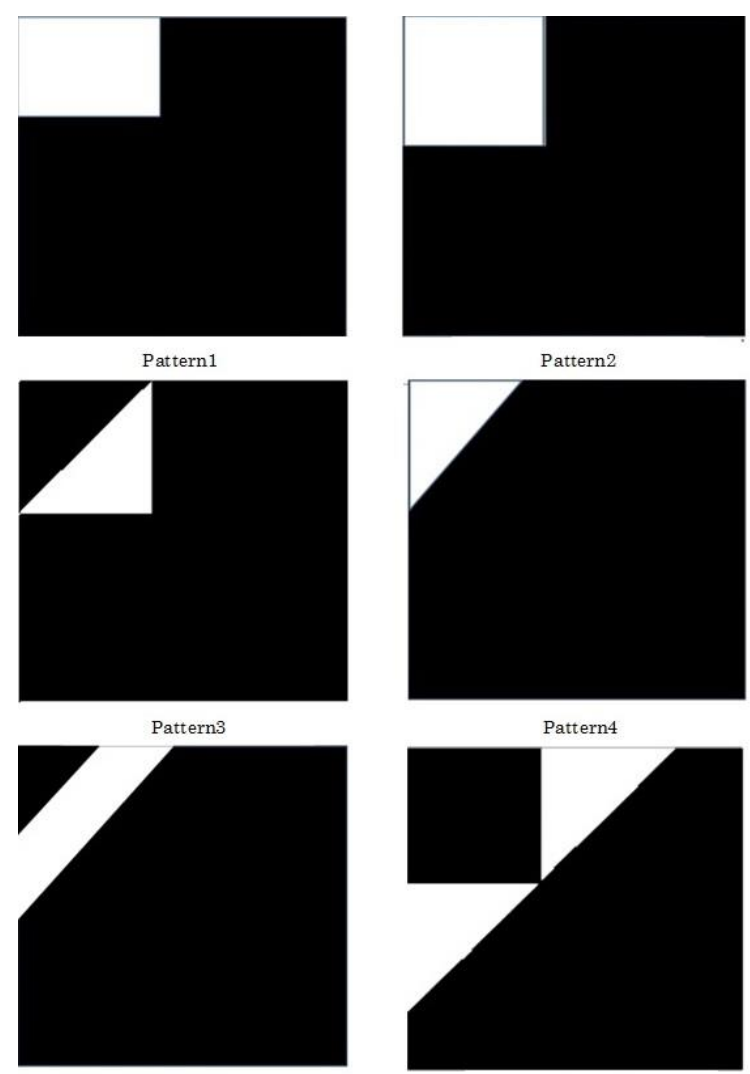

Pattern5

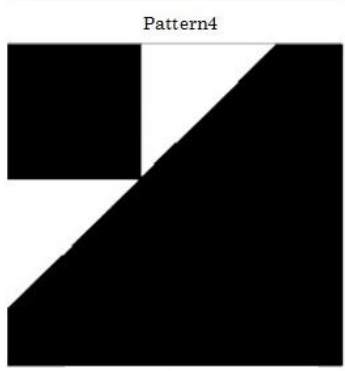

Pattern6

Fig.1. Shapes to use.

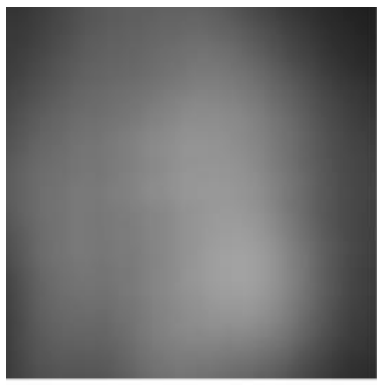

$351 \times 351$

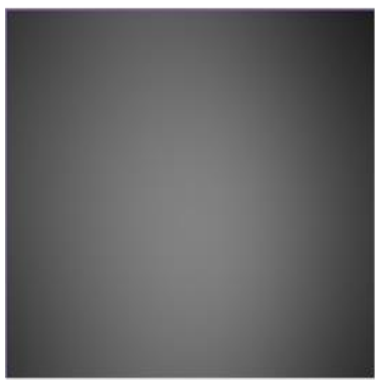

$751 \times 751$

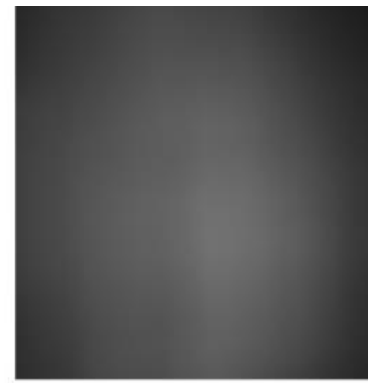

$501 \times 501$

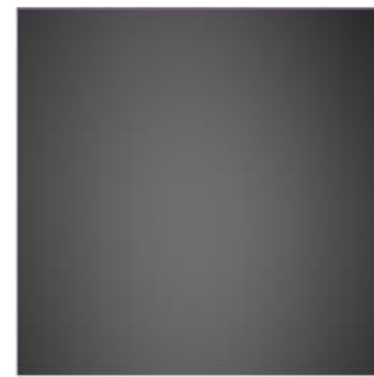

$1001 \times 1001$
Fig.2. Luminance Distribution.

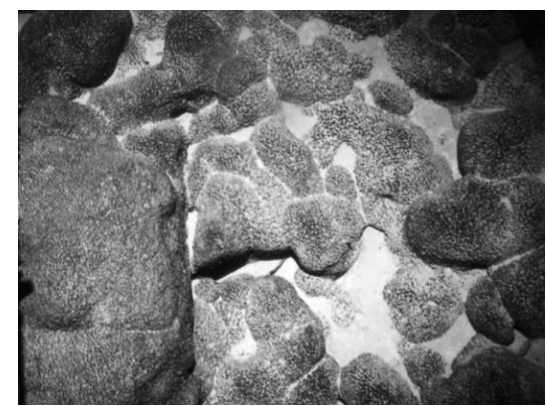

Fig.3. Original Image.

\section{Result}

The image after the $\mathrm{YCrCb}$ conversion is as shown in Fig.3. The result of the proposed method is as shown in Fig.4. The result of the conventional method is as shown in Fig.5.

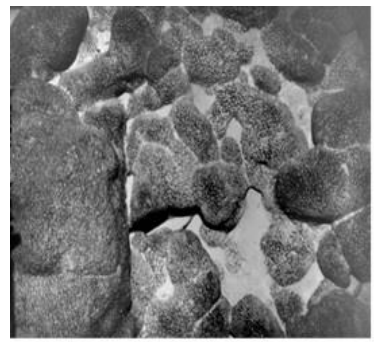

Pattern1

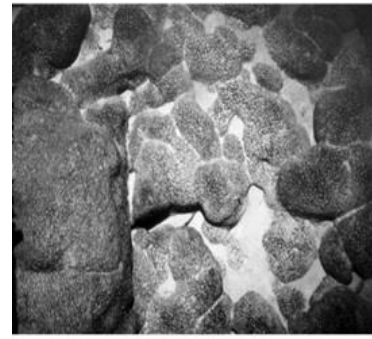

Pattern3

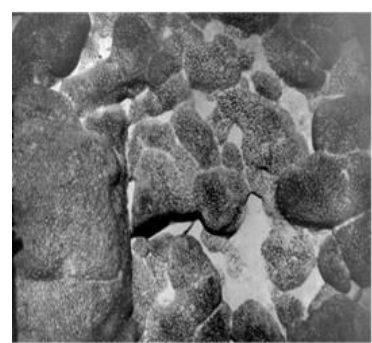

Patterñ̄

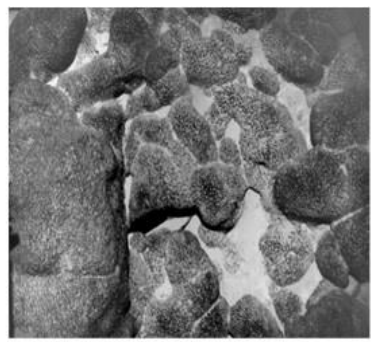

Pattern2

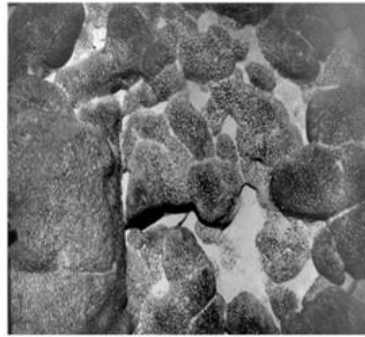

Pattern 4

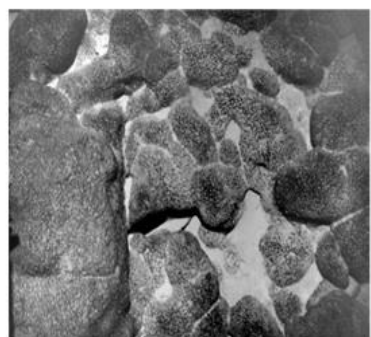

Pattern6
Fig.4. Proposed Result. 

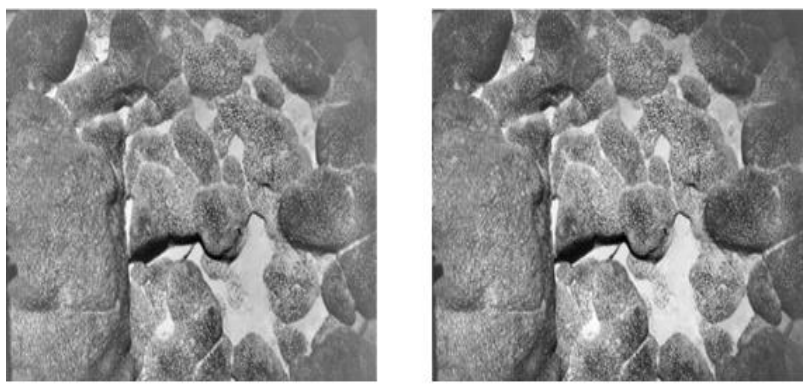

$351 \times 351$

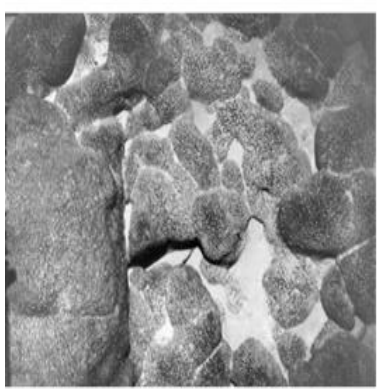

$751 \times 751$
$501 \times 501$

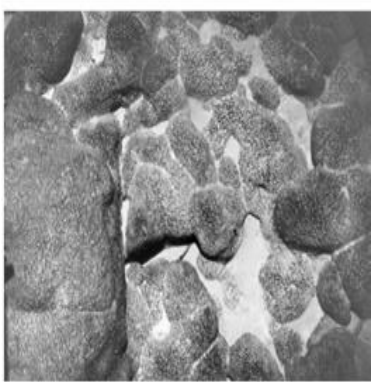

$1001 \times 1001$

Fig.5. Conventional Method.

\section{Conclusions}

As shown in Fig.4, the luminance of the whole image was equalized by the proposed method. Among the DCT coefficients in the low frequency region, there are components that do not greatly affect the uniformity of the luminance. Pattern 3 in Fig. 4 is an example. Therefore, I want to find the optimal solution of the region where the DCT component necessary to equalize the luminance of the whole image is 0 .

\section{References}

(1) JAMSTEC : Undersea Research and Development Center,

https://www.jamstec.go.jp/shigen/j/exploration/shi gen.html

(2) GODAC : International Marine Environment Information Center,

http://www.godac.jp/education/deepsea.html

(3) Shin Kisugi, Isao Koike and Hiroshi Terashima :

"Introduction to ocean problems",2007

(4) Gary Bradski and Adrian Kaehler : "Learning OpenCV",2008

(5) K.R. Rao and P.Yip : "Discrete Cosine Transform: Algorithms, Advantages, Applications”, 1990 